\title{
Two Particular GV-semigroups with 0-semidistributive Full Subsemigroup Lattices
}

\author{
Yu Wang and Yong Yu \\ Department of Mathematics, Anhui University of Science and Technology, Huainan, Anhui 232001, P. R. China \\ Email: wangyu4440185@126.com
}

\begin{abstract}
The aim of this paper is to study two particular GV-semigroups whose full subsemigroups form 0-semidistributive lattices. The structure of GV-inverse semigroups whose full subsemigroup lattices are 0 -semidistributive is first characterized. Moreover, much more explicit structural description is obtained for orthodox GV-semigroups by establishing an inverse semigroup congruence.
\end{abstract}

Keywords: GV-semigroup, full subsemigroup lattice, 0-semidistributive lattice.

\section{Introduction}

The subsemigroup lattices of semigroups have been the subject of continued investigation for many years. The main achievements in the area, accomplished by the mid 1990s, have been comprehensively reflected in the monograph [14]. Among the large fields of the investigation, much attention has been paid to the full regular subsemigroup lattices of regular semigroups. Recall that a subset of a semigroup is called full if it contains the set of all idempotents of the given semigroup.

Johnston and Jones researched regular semigroups with their full regular subsemigroup lattices in [7], which provides some interesting results. It is well known that full regular subsemigroups of inverse semigroups are just their full inverse subsemigroups. Thus the theory concerning full regular subsemigroup lattices has been extensively explored in the case of inverse semigroups. A series of papers have been devoted to the theme of describing the structure of inverse semigroups with various types of full regular subsemigroup lattices (see $[8,9,10,11,12,16]$ ). Moreover, Jones and Tian generalized to eventually regular semigroups the study of the full regular subsemigroup lattices of regular semigroups and characterized the structure of eventually regular semigroups whose full eventually regular subsemigroup lattices are distributive lattices or chains in [13]. Recently, a programme of studying the interrelationships of inverse semigroups and their full subsemigroup lattices has been presented in [15] by Tian, who established the structure of inverse semigroups with various assumptions on their full subsemigroup lattices.

\section{Preliminaries}

Recall that a lattice $L$ with zero is called 0 -semidistributive, if for any $a, b, c \in L, a \wedge b=a \wedge c=0$ implies $a \wedge(b \vee c)=0$. A semigroup $S$ is called eventually regular if some power of each element of $S$ is regular. If every regular element of an eventually regular semigroup $S$ is completely regular, then $S$ is called a $G V$-semigroup. For a completely regular semigroup $S$, every regular element $a$ of $S$ exists and only exists an inverse of $a$ which commutes with $a$. We usually denote the unique inverse of $a$ by $a^{-1}$. If every regular element of an eventually regular semigroup $S$ possesses a unique inverse, then $S$ is called eventually inverse, and the unique inverse of $a \in S$ is denoted by $a^{-1}$. A semigroup $S$ is called orthodox [eventually orthodox] if it is a regular [ an eventually regular] semigroup whose the set $E_{S}$ forms a subsemigroup of $S$. In particular, a GV-semigroup is called a $G V$-inverse semigroup [orthodox $G V$-semigroup ] if it is eventually inverse [eventually orthodox]. For any $a \in S$, an element $x$ of $S$ is called a weak inverse of $a$ if $x a x=x$. Denote by $W(a)$ the set of all weak inverses of $a$ in $S$, and by $V(a)$ the set of all inverses of $a$ in $S$.

For any subset $A$ of a semigroup $S$, we denote by $\langle A\rangle$ the subsemigroup of $S$ generated by $A$, by $A^{*}$ the set of all non-zero elements of $A$, by $\operatorname{Sub} S$ the lattice of all subsemigroups (including the empty set) of $S$, and by Subf $S$ the lattice of all full subsemigroups of $S$. It is easy to show that the lattice Subf $S$ is a 
complete sublattice of the lattice SubS. In particular, for an inverse semigroup $S$, we shall denote by Subfi $S$ the lattice of full inverse subsemigroups of $S$ as in $[8,9]$. If $\mathcal{K}$ is one of the Green's relations $\mathcal{L}, \mathcal{R}$, $\mathcal{H}, \mathcal{D}$ and $\mathcal{J}$ on a semigroup $S$, then we will use $K_{a}$ to denote the $\mathcal{K}$-class of $S$ containing an element $a$ in $S$, and use $\mathcal{K}^{A}$ to denote the relation $\mathcal{K}$ restricting to a subsemigroup $A$ of $S$.

A semigroup $S$ is called to be combinatorial if its subgroups are all trivial. In this case, it is easy to observe that each $\mathcal{H}$-class of $S$ has exactly one element if $S$ is regular. An inverse semigroup $S$ is called to be E-unitary if $e x, e \in E_{S}$ implies $x \in E_{S}$, or equivalently if $\mathcal{R} \cap \sigma=1$, where $\sigma=\{(a, b) \in S \times S: e a=$ $e b$ for some $\left.e \in E_{S}\right\}$ is the minimal group congruence on $S$. For an inverse semigroup $S$ that is E-unitary, it is obvious that the set defined by $\operatorname{ker} \sigma=\left\{x \in S: x \sigma e\right.$ for some $\left.e \in E_{S}\right\}$ is equal to $E_{S}$. A completely 0 -simple inverse semigroup is called Brandt semigroup. In Section 3, we need consider a combinatorial Brandt semigroup $B_{5}=<a, b \mid a b a=a, b a b=b, a^{2}=b^{2}=0>$ with exactly two non-zero idempotents and two non-idempotents.

Let $J$ be any $\mathcal{J}$-class of a semigroup $S$. We shall define the principal factor $P F(J)$ of $S$ as the set $J$ with a zero adjoined, the product of two elements of $J$ being their product in $S$ if it lies in $J$, and all other products being zero. Here, the zero should be adjoined to $J$ even if $J$ is a minimal $\mathcal{J}$-class of $S$. It is well known that any principal factor of a semigroup is either null (that is, the product of any two elements is zero) or 0-simple semigroup. Note that the present definition of principal factor varies slightly from the traditional one in [2], in which the definition is that $P F(J)=J$ if $J$ is minimal and $P F(J)=J \cup\{0\}$ if $J$ is not minimal.

The following lemma provides an important property for the $\mathcal{J}$-classes of eventually regular semigroups.

Lemma 2.1. ([13], Proposition 1.5) Let $S$ be an eventually regular semigroup and let $J$ be a $\mathcal{J}$-class of $S$ that contains an idempotent. Then every element of $J$ is regular. Equivalently, any 0-simple eventually regular semigroup is regular.

It follows from Lemma 2.1 that a product being irregular lies in a $\mathcal{J}$-class that must consist entirely of irregular elements. For a $\mathrm{GV}$-semigroup, we classify $\mathcal{J}$-class as either irregular or regular and its principal factors are either null or regular.

Lemma 2.2. ([17], Corollary 3.3 and Proposition 3.7) Let $S$ be an E-semigroup. Then

(1) $W(a b)=W(b) W(a)$ and $W(e) \subseteq E_{S}$ for any $a, b \in S, e \in E_{S}$;

(2) $W(a)=E_{S} a^{\prime} E_{S}$ for any $a \in \operatorname{Reg} S, a^{\prime} \in V(a)$.

By virtue of Lemma 2.2 we shall give another characterization on weak inverses in an E-semigroup.

Lemma 2.3. Let $S$ be an E-semigroup. Then

(1) $W(e)=W(f)$ for some $e, f \in E_{S} \Longleftrightarrow e \mathcal{J}^{E_{S}} f$;

(2) $W(a)=W\left(a^{\prime} a\right) a^{\prime} W\left(a a^{\prime}\right)$ for any $a \in \operatorname{Reg} S$, $a^{\prime} \in V(a)$.

Proof. To prove " $\Longrightarrow$ ", suppose that $W(e)=W(f)$ for some $e, f \in E_{S}$. It is obvious that $e, f \in \operatorname{Reg} S$ and $e \in V(e), f \in V(f)$. It follows from Lemma 2.2 that $W(e)=E_{S} e E_{S}=E_{S} f E_{S}=W(f)$. Hence $e \in W(e)=E_{S} f E_{S}, f \in W(f)=E_{S} e E_{S}$, and so $e \mathcal{J}_{E_{S}} f$. We now show "£". Suppose that $e \mathcal{J}_{E_{S}} f$ for some $e, f \in E_{S}$. Then there exist $e_{1}, e_{2}, f_{1}, f_{2} \in E_{S}$ such that $e=e_{1} f e_{2}, f=f_{1} e f_{2}$. By Lemma 2.2 again, it follows that $W(e)=E_{S} e E_{S}=E_{S} e_{1} f e_{2} E_{S} \subseteq E_{S} f E_{S}=W(f)$, which shows that $W(e) \subseteq W(f)$. A similar argument will show that $W(f) \subseteq W(e)$. Hence $W(e)=W(f)$, and so part (1) has been proved.

We then turn to proving part (2). It follows from Lemma 2.2 that $W(a)=E_{S} a^{\prime} E_{S}$ and $W\left(a^{\prime} a\right), W\left(a a^{\prime}\right) \subseteq$ $E_{S}$ for any $a \in \operatorname{Reg} S, a^{\prime} \in V(a)$. Therefore $W\left(a^{\prime} a\right) a^{\prime} W\left(a a^{\prime}\right) \subseteq E_{S} a^{\prime} E_{S}=W(a)$. To prove the converse inclusion, take any $a^{*} \in W(a)$. Then $a^{*}=a^{*} a a^{*}=\left(a^{*} a\right) a^{\prime}\left(a a^{*}\right)$. Notice that

$$
\left(a^{*} a\right) a^{\prime} a\left(a^{*} a\right)=a^{*} a a^{*} a=a^{*} a,\left(a a^{*}\right) a a^{\prime}\left(a a^{*}\right)=a a^{*} a a^{*}=a a^{*},
$$

and so $a^{*} a \in W\left(a^{\prime} a\right), a a^{*} \in W\left(a a^{\prime}\right)$. Hence $a^{*}=\left(a^{*} a\right) a^{\prime}\left(a a^{*}\right) \subseteq W\left(a^{\prime} a\right) a^{\prime} W\left(a a^{\prime}\right)$, so that $W(a) \subseteq$ $W\left(a^{\prime} a\right) a^{\prime} W\left(a a^{\prime}\right)$. Consequently, we obtain that $W(a)=W\left(a^{\prime} a\right) a^{\prime} W\left(a a^{\prime}\right)$ for any $a \in \operatorname{Reg} S, a^{\prime} \in$ $V(a)$. 


\section{0-semidistributivity in the GV-inverse semigroup}

In this section, we shall give a structure theorem for GV-inverse semigroups with 0-semidistributive full subsemigroup lattices. It follows from the definition that the set of all regular elements of a GV-inverse semigroup is an inverse subsemigroup. Therefore we first characterize inverse semigroups whose full subsemigroup lattice is 0 -semidistributive.

The following lemma is a generalization of Lemma 3.2 in [15].

Lemma 3.1. Let $S$ be an inverse semigroup. Then the map $\Sigma$ defined by $A \rightarrow A \Sigma=\{a \sigma: a \in A\}$ for any $A \in \operatorname{Subf} S$, where $\sigma$ is the minimum group congruence on $S$, is a surjective lattice homomorphism of Subf $S$ upon $\operatorname{Subf}(S / \sigma)$ that preserves complete join.

Proof. Let $A, B \in \operatorname{Subf} S$. Then it is straightforward to show that $(A \vee B) \Sigma=A \Sigma \vee B \Sigma$. It is clear that $A \Sigma \cap B \Sigma \supseteq(A \cap B) \Sigma$. To prove the converse inclusion, let $x \in A \Sigma \cap B \Sigma$. Then there exist $a \in A$ and $b \in B$ such that $x=a \sigma=b \sigma$, and so $e a=e b, a^{-1} e=b^{-1} e$ and $b a^{-1} e=b b^{-1} e \in E_{S}$ for some $e \in E_{S}$, hence $\left(b a^{-1} e\right) a \in A$. On the other hand, it follows from $a^{-1} e a \in E_{S}$ that $b\left(a^{-1} e a\right) \in B$, and so $b a^{-1} e a \in A \cap B$. Notice that $x=b \sigma=\left(b\left(a^{-1} e a\right)\right) \sigma \in(A \cap B) \sigma$, that is, $A \Sigma \cap B \Sigma \subseteq(A \cap B) \Sigma$. Hence $A \Sigma \cap B \Sigma=(A \cap B) \Sigma$, and so the map $\Sigma$ is a lattice homomorphism.

We now verify that the homomorphism $\Sigma$ is surjective. Let $U \in \operatorname{Subf}(S / \sigma)$ and put $A=\{s \in S$ : $s \sigma \in U\}$. Since $E_{S} \subseteq A$ and $(a b) \sigma=a \sigma b \sigma \in U$ for any $a, b \in A$, we have $a b \in A$ and $A \Sigma=U$, where $A \in \operatorname{Subf} S$. Therefore the homomorphism $\Sigma$ is surjective. The fact that the homomorphism $\Sigma$ preserves complete join is obvious.

Lemma 3.2. For a non-trivial group $G$ with the identity e, the lattice $\operatorname{Subf} G$ is 0-semidistributive if and only if for any $a_{1}, a_{2}, \ldots, a_{n} \in G$ with $a_{1} a_{2} \cdots a_{n} \neq e$, there exists $a_{t}$ such that $\left(a_{1} a_{2} \cdots a_{n}\right)^{m} \in\left\langle a_{t}\right\rangle \backslash\{e\}$ for some $m \in Z^{+}$(the set of positive integers).

Proof. Put $a=a_{1} a_{2} \cdots a_{n}$, and suppose that $\langle e, a\rangle \cap\left\langle e, a_{i}\right\rangle=\{e\}$ for each $i=1,2, \ldots, n$. Then by 0 -semidistributivity of the lattice $\operatorname{Subf} G$, we have $\langle e, a\rangle \cap\left\langle e, a_{1}, a_{2}\right\rangle=\{e\}$. It is easy to obtain that $\langle e, a\rangle \cap\left\langle e, a_{1}, a_{2}, \ldots, a_{n}\right\rangle=\{e\}$, contradicting to the assumption that $a=a_{1} a_{2} \cdots a_{n} \neq e$. Hence there exist $b \in\left(\langle e, a\rangle \cap\left\langle e, a_{t}\right\rangle\right) \backslash\{e\}$ for some $a_{t}$, and so $a^{m}=b \in\left\langle a_{t}\right\rangle \backslash\{e\}$ for some $m \in Z^{+}$.

Conversely, let $A, B, C \in \operatorname{Subf} G$ with $A \cap B=A \cap C=\{e\}$. Suppose that there exists $a \in G \backslash\{e\}$ such that $a \in A \cap(B \vee C)$. Then $a=a_{1} a_{2} \cdots a_{n}$, where each $a_{i} \in B \cup C, i=1,2, \ldots, n$, whence there exists some $a_{t}$ such that $a^{m} \in\left\langle a_{t}\right\rangle \backslash\{e\} \subseteq B \cup C$ for some $m \in Z^{+}$, together with $a^{m} \in A$, and so $a^{m} \in A \cap(B \cup C)=\{e\}$, which leads to a contradiction. Therefore $A \cap(B \vee C)=\{e\}$, which shows that the lattice $\operatorname{Subf} G$ is 0 -semidistributive.

Using Corollary 2.2 in [16] and Lemma 3.2, we have the following

Corollary 3.3. For a torsion-free group $G$ with the identity e, the following conditions are equivalent:

(1) Subf $G$ is 0-semidistributive ;

(2) SubfiG is 0-semidistributive;

(3) For any $a, b \in G$ with $a b \neq e$, there exist $m, n \in Z^{+}$such that $(a b)^{m}=a^{n}$ or $b^{n}$.

Let $X$ be a set. As in [15], we denote by $\mathcal{R} \mathcal{T}(X)$ the set of binary relations satisfying reflexivity and transitivity on $X$. It is obvious that $\mathcal{R} \mathcal{T}(X)$ is a complete lattice. Suppose that $|X| \geq 3$, and let $x_{1}, x_{2}, x_{3}$ be distinct elements of $X$. Put $\rho_{1}=\left\{\left(x_{1}, x_{2}\right),\left(x_{1}, x_{3}\right)\right\} \cup\{(x, x): x \in X\}, \rho_{2}=\left\{\left(x_{2}, x_{3}\right)\right\} \cup\{(x, x): x \in X\}$ and $\rho_{3}=\left\{\left(x_{2}, x_{1}\right)\right\} \cup\{(x, x): x \in X\}$. Clearly, $\rho_{1}, \rho_{2}, \rho_{3} \in \mathcal{R} \mathcal{T}(X)$. Notice that $\rho_{2} \cap \rho_{1}=\rho_{2} \cap \rho_{3}=1_{X}$ and

$$
\rho_{1} \vee \rho_{3}=\left\{\left(x_{1}, x_{2}\right),\left(x_{1}, x_{3}\right),\left(x_{2}, x_{3}\right),\left(x_{2}, x_{1}\right)\right\} \cup\{(x, x): x \in X\} .
$$

Hence $\rho_{2} \subseteq \rho_{1} \vee \rho_{3}$, and so $\rho_{2} \cap\left(\rho_{1} \vee \rho_{3}\right)=\rho_{2} \neq 1_{X}$. It follows that the lattice $\mathcal{R} \mathcal{T}(X)$ is not 0 -semidistributive. Combining with Lemma 3.7 in [15], we then have the following

Lemma 3.4. For any set $X$, the following conditions are equivalent:

(1) $\mathcal{R} \mathcal{T}(X)$ is distributive ;

(2) $\mathcal{R} \mathcal{T}(X)$ is 0-semidistributive ;

(3) $|X| \leq 2$. 
It suffices to consider 0 -semidistributivity for 0 -simple inverse semigroups when we investigate inverse semigroups whose full subsemigroups form 0-semidistributive lattices. Therefore we consider first completely 0 -simple inverse semigroups, that is, 0 -simple inverse semigroups containing a primitive idempotent.

Lemma 3.5. For a Brandt semigroup $S$, the lattice Subf $S$ is 0-semidistributive if and only if either $S$ is isomorphic to $B_{5}$, or $S^{*}$ is a group whose lattice Subf $S$ is 0-semidistributive.

Proof. Let $S$ be a Brandt semigroup, whose lattice Subf $S$ is 0 -semidistributive. Assume first that $\left|E_{S}^{*}\right|=1$. Then $S^{*}$ is a group, and so Subf $S \cong \operatorname{Subf} S^{*}$. Therefore the lattice Subf $S^{*}$ is 0 -semidistributive.

Suppose now that $\left|E_{S}^{*}\right|>1$. Note that the lattice Subfi $S$ is 0 -semidistributive since the lattice SubfiS is a complete sublattice of the lattice Subf $S$. Therefore it follows from Theorem 2.3 in [16] that $S$ is combinatorial, and so $S$ is a combinatorial Brandt semigroup. Following Theorem 3.8 in [15], stating that for a combinatorial Brandt semigroup $S$, Subf $S \cong \mathcal{R} T\left(E_{S}^{*}\right)$, we, by Lemma 3.4, deduce that $\left|E_{S}^{*}\right|=2$. Thus $S$ is isomorphic to $B_{5}$.

The sufficiency follows from Theorem 3.8 in [15], Lemmas 3.2 and 3.4.

We now turn to considering 0-simple inverse semigroups that are not completely 0-simple cases. Referring to Lemma 2.4 in [16] we know that if $S$ is a non-completely 0-simple inverse semigroup whose lattice Subfi $S$ is 0 -semidistributive, then $S$ has no zero divisor and Subfi $S \cong \operatorname{Subfi}\left(S^{*}\right)$. Therefore it remains to consider the full subsemigroup lattices of simple inverse semigroups that is not groups.

Lemma 3.6. For a simple inverse semigroup $S$ that is not a group, the lattice Subf $S$ is 0-semidistributive if and only if $S$ is E-unitary, combinatorial and for $a, b \in S / \sigma$ with ab $\neq e$, there exist $m, n \in Z^{+}$such that $(a b)^{m}=a^{n}$ or $b^{n}$.

Proof. Suppose that a simple inverse semigroup $S$ (not a group), whose lattice Subf $S$ is 0-semidistributive. Then the lattice SubfiS is 0-semidistributive, and so the necessity follows from Theorem 2.10 in [16] immediately.

Conversely, let $A, B, C \in \operatorname{Subf} S$ with $A \cap B=A \cap C=E_{S}$. Suppose that there exists $a \in S \backslash E_{S}$ such that $a \in A \cap(B \vee C)$. Since the map $\Sigma$ induced by $\sigma$ is a surjective homomorphism of Subf $S$ upon $\operatorname{Subf}(S / \sigma)$ by Lemma 3.1, we have $A \Sigma \cap B \Sigma=A \Sigma \cap C \Sigma=\{e\}$ and $a \sigma \in A \Sigma \cap(B \Sigma \vee C \Sigma)$, where $a \sigma \neq e$ by the fact that $S$ is E-unitary. On the other hand, the lattice $\operatorname{Subf}(S / \sigma)$ is 0 -semidistributive according to Lemma 2.7 in [16] and Corollary 3.3. Therefore $A \Sigma \cap(B \Sigma \vee C \Sigma)=\{e\}$ by the 0-semidistributivity of the lattice $\operatorname{Subf}(S / \sigma)$, which contradicts to $e \neq a \sigma \in A \Sigma \cap(B \Sigma \vee C \Sigma)$. Consequently, $A \cap(B \vee C)=E_{S}$, which shows that the lattice Subf $S$ is 0-semidistributive.

Now we are ready for the main result in this section.

Theorem 3.7. For a GV-inverse semigroup $S$ that is not inverse, if the lattice $\operatorname{Subf} S$ is 0-semidistributive then

(1) each regular $\mathcal{J}$-class containing a primitive idempotent is either a group whose the lattice Subf $S$ is 0 -semidistributive, or has principal factor isomorphic to $B_{5}$; and

(2) each regular $\mathcal{J}$-class not containing a primitive idempotent is E-unitary, combinatorial, and for $a, b \in S / \sigma$ with $a b \neq e$, there exist $m, n \in Z^{+}$such that $(a b)^{m}=a^{n}$ or $b^{n}$.

Proof. Let $S$ be a GV-inverse semigroup. Then the set $\operatorname{Reg} S$ is an inverse subsemigroup of $S$. Hence the lattice $\operatorname{Subf}(\operatorname{Reg} S)$ coincides with the interval $\left[E_{S}, \operatorname{Reg} S\right]$ of the lattice Subf $S$, which is 0-semidistributive, and so the lattice $\operatorname{Subf}(\operatorname{Reg} S)$ is 0 -semidistributive. It then follows that the lattice $\operatorname{Subf}(P F(J))$ is 0 -semidistributive for each $\mathcal{J}$-class $J$ of the set $\operatorname{Reg} S$. Note that each regular $\mathcal{J}$-class $J$ of $S$ turns out to be $\mathcal{J}$-class of $\operatorname{Reg} S$. Therefore conditions (1) and (2) follow from Lemma 3.5 and 3.6.

\section{0-semidistributivity in the orthodox GV-semigroup}

For an orthodox $\mathrm{GV}$-semigroup $S$, it is well known that $E_{S}$ is a semilattice of rectangular bands (that is, simple bands). In precise, components of $E_{S}$ in this decomposition are just its $\mathcal{J}_{E_{S}}$-classes. For convenience, we shall denote $\mathcal{J}_{E_{S}}$ by $\pi$, and $E_{S}^{*} / \pi$ by $\varepsilon(S)$ in the rest of this section. By virtue of the statement in the paragraph following Lemma 2.1, we obtain that $P F(J)$ is a 0 -simple orthodox semigroup for each regular $\mathcal{J}$-class $J$ of $S$. 
Lemma 4.1. Let $S$ be an orthodox $G V$-semigroup and let $J$ be any regular $\mathcal{J}$-class $J$ of $S$. Then the relation $\gamma$ defined by $\gamma=\{(a, b) \in S \times S: W(a)=W(b)\}$ is a congruence on $S$. Furthermore, the relation $\gamma$, restricting to $P F(J)$, is an inverse semigroup congruence and $\operatorname{ker} \gamma=E_{P F(J)}$.

Proof. It is a routine matter to show that the relation $\gamma$ is an equivalence on $S$. Let $a \gamma b$, that is, $W(a)=W(b)$ for some $a, b \in S$. Since $S$ is eventually regular, each element of $S$ exists weak inverse. Take any $c \in S, t \in W(c a)$. Then $t \in W(c a)=W(a) W(c)$ by Lemma 2.2, and so there exist $a^{\prime} \in W(a)=$ $W(b), c^{\prime} \in W(c)$ such that $t=a^{\prime} c^{\prime} \in W(b) W(c)=W(c b)$, so that $W(c a) \subseteq W(c b)$. Dually, we may show

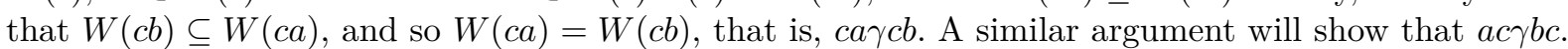
Thus the relation $\gamma$ is a congruence on $S$.

To prove the latter assertion, let $J$ be any regular $\mathcal{J}$-class $J$ of $S$. Then $P F(J)$ is a 0 -simple orthodox semigroup, and so the quotient $P F(J) / \gamma$ is regular. It then follows from Lallement's Lemma and Lemma 2.3 that $E_{(P F(J) / \gamma)}$ is a semilattice. Therefore $P F(J) / \gamma$ is an inverse semigroup, which shows that the congruence $\gamma$ is an inverse semigroup congruence when $\gamma$ restricts to $\operatorname{PF}(J)$. We shall prove that $\operatorname{ker} \gamma=E_{P F(J)}$. Assume that there exists $b \in P F(J) \backslash E_{P F(J)}$ and $e \in E_{P F(J)}^{*}$ such that $b \gamma e$. Then $W(b)=W(e) \subseteq E_{P F(J)}^{*}$. Further, since $P F(J)$ is a regular semigroup, we have $V(b) \subseteq W(b)=W(e) \subseteq$ $E_{P F(J)}^{*}$, and so there exists $f \in E_{P F(J)}^{*}$ such that $f b f=f, b f b=b$. Hence $b \in V(f) \subseteq W(f) \subseteq E_{P F(J)}^{*}$, which contradicts the assumption. Therefore $\operatorname{ker} \gamma=E_{P F(J)}$, as required.

Lemma 4.2. Let $S$ be a 0-simple orthodox semigroup. Then the map $\Gamma$ defined by $A \rightarrow A \Gamma=\{a \gamma: a \in A\}$ for any $A \in \operatorname{Subf} S$, where $\gamma$ is the relation defined in Lemma 4.1, is a lattice isomorphism from Subf $S$ upon $\operatorname{Subf}(S / \gamma)$.

Proof. Let $A, B \in \operatorname{Subf} S$. Then it is straightforward to show that $(A \vee B) \Gamma=A \Gamma \vee B \Gamma$ and $A \Gamma \cap B \Gamma \supseteq$ $(A \cap B) \Gamma$. To prove the converse inclusion, let $x \in A \Gamma \cap B \Gamma$. Then there exist $a \in A$ and $b \in B$ such that $x=a \gamma=b \gamma$. Since the relation $\gamma$ is a congruence on $S$ proved in Lemma 4.1, we have $a^{\prime} a \gamma a^{\prime} b$ for any $a^{\prime} \in W(a)$. It follows, by the property that ker $\gamma=E_{S}$ showed in Lemma 4.1, that $a^{\prime} b \in E_{S}$ and $a\left(a^{\prime} b\right) \in A,\left(a a^{\prime}\right) b \in B$, where $A, B \in \operatorname{Subf} S$. Hence $a a^{\prime} b \in A \cap B$. Note that $\gamma$ is a regular congruence on $S$. Then we, according to Lemma 5.4 in [18], know that there exists $a^{\prime \prime} \in W(a)$ such that $a \gamma a a^{\prime \prime} a$, and so $x=a \gamma=a a^{\prime \prime} a \gamma=a a^{\prime \prime} b \gamma$. And since $a a^{\prime} b \in A \cap B$ for any $a^{\prime} \in W(a)$, it follows that $x \in(A \cap B) \Gamma$, and so $A \Gamma \cap B \Gamma \subseteq(A \cap B) \Gamma$. Therefore $A \Gamma \cap B \Gamma=(A \cap B) \Gamma$, which gives that the map $\Gamma$ is a lattice homomorphism.

To show that the homomorphism $\Gamma$ is surjective, let $U \in \operatorname{Subf}(S / \gamma)$, and put $A=\{s \in S: s \gamma \in U\}$. Since $E_{S} \subseteq A$ and $(a b) \gamma=a \gamma b \gamma \in U$ for any $a, b \in A$, it follows that $a b \in A$ and $A \Gamma=U$, where $A \in$ Subf $S$. Therefore the homomorphism $\Gamma$ is surjective. We finally show that $\Gamma$ is injective. Let $A, B \in \operatorname{Subf} S$ such that $A \Gamma=B \Gamma$. Take any $a \in A$. Then there exists $b \in B$ such that $a \gamma=b \gamma$, that is,$W(a)=W(b)$. Hence for any $a^{\prime} \in V(a) \subseteq W(a)=W(b)$, we have $a^{\prime} b a=a^{\prime}, a=a a^{\prime} a=a\left(a^{\prime} b a^{\prime}\right) a=\left(a a^{\prime}\right) b\left(a^{\prime} a\right) \in B$, and so $A \subseteq B$. Similarly, we may show that $B \subseteq A$. Therefore $A=B$. Up to now, we have proved that $\Gamma$ is a lattice isomorphism from $\operatorname{Subf} S$ upon $\operatorname{Subf}(S / \gamma)$.

As the title of this section indicated, we are to establish the structure for orthodox GV-semigroups. Let $S$ be a orthodox GV-semigroup. It is easy to observe that $P F(J)$ is completely 0-simple for each regular $\mathcal{J}$-class $J$ of $S$. We thus only need to consider $P F(J)$ by two cases, that is, combinatorial completely 0 -simple case or non-combinatorial completely 0-simple case.

Lemma 4.3. For a combinatorial completely 0-simple orthodox semigroup $S$, the lattice Subf $S$ is 0 semidistributive if and only if $S$ is either a 0 -simple band or $|\varepsilon(S)|=2$.

Proof. We show first that the quotient $S / \gamma$ is a combinatorial completely 0 -simple inverse semigroup. It follows by Lemma 4.1 that $S / \gamma$ is inverse. To prove that $S / \gamma$ is completely 0 -simple, let any $a \gamma, b \gamma \in S / \gamma$ for some $a, b \in S^{*}$. Since $S$ is 0-simple, there exist $x_{1}, y_{1}, x_{2}, y_{2} \in S$ such that $a=x_{1} b y_{1}, b=x_{2} a y_{2}$. Therefore $a \gamma=\left(x_{1} \gamma\right)(b \gamma)\left(y_{1} \gamma\right), b \gamma=\left(x_{2} \gamma\right)(a \gamma)\left(y_{2} \gamma\right)$, and so $S / \gamma$ is 0-simple. By virtue of the property that $S$ is complete, we know that $S$ at least contains a primitive idempotent $e$. Then it is easy to check that $e \gamma$ is a primitive idempotent of $S / \gamma$, and so $S / \gamma$ is a completely 0-simple semigroup.

To prove that the quotient $S / \gamma$ is combinatorial, assume that $S$ is not combinatorial. Then there exist $a, b \in S$ such that $a \gamma \neq b \gamma$ and $(a \gamma) \mathcal{H}(b \gamma)$. Since $S / \gamma$ is inverse, we may denote by $(a \gamma)^{-1}$ and 
$(b \gamma)^{-1}$ the unique inverses of $a \gamma$ and $b \gamma$, respectively. It is obvious that $a^{\prime} \gamma=(a \gamma)^{-1}$ and $b^{\prime} \gamma=(b \gamma)^{-1}$ for any $a^{\prime} \in V(a), b^{\prime} \in V(b)$. It follows from $(a \gamma) \mathcal{H}(b \gamma)$ that $a \gamma(a \gamma)^{-1}=\left(a a^{\prime}\right) \gamma=\left(b b^{\prime}\right) \gamma=b \gamma(b \gamma)^{-1}$ and $(a \gamma)^{-1} a \gamma=\left(a^{\prime} a\right) \gamma=\left(b^{\prime} b\right) \gamma=(b \gamma)^{-1} b \gamma$. On the other hand, since $S$ is completely 0-simple, it follows that $b \mathcal{R} b\left(b^{\prime} a b^{\prime} b\right)$ and $b \mathcal{L}\left(b b^{\prime} a b^{\prime}\right) b$. Hence $b \mathcal{H} b b^{\prime} a b^{\prime} b$, and so $b=b b^{\prime} a b^{\prime} b$ from the fact that $S$ is combinatorial. And by $(a a) \gamma=\left(b^{\prime} b\right) \gamma$ and $\left(a a^{\prime}\right) \gamma=\left(b b^{\prime}\right) \gamma$ proved above, notice that

$$
a \gamma=a a^{\prime} a \gamma=\left(a a^{\prime} b b^{\prime}\right) a a^{\prime} a \gamma=a a^{\prime}\left(b b^{\prime} a b^{\prime} b\right) a^{\prime} a \gamma=b b^{\prime} b a^{\prime} a \gamma=b b^{\prime} b b^{\prime} b \gamma=b \gamma,
$$

which leads to a contradiction. Therefore $S / \gamma$ is combinatorial, and so $S / \gamma$ is a combinatorial completely 0 -simple inverse semigroup, as required. From Theorem 3.8 in [15] and Lemma 4.2 we obtain that $\operatorname{Subf} S \cong \operatorname{Subf}(S / \gamma) \cong \mathcal{R} T\left(E_{S / \gamma}^{*}\right)$, and so $\left|E_{S / \gamma}^{*}\right| \leq 2$ by Lemma 3.4. It follows, by the property that ker $\gamma=E_{S}$ and Lemma 2.3, that $E_{S / \gamma}^{*}=E_{S}^{*} / \gamma=E_{S}^{*} / \pi=\varepsilon(S)$. Thus $|\varepsilon(S)|=\{1,2\}$. If $|\varepsilon(S)|=1$, then it is easy to see that $S$ is a 0 -simple band. In fact, it follows from $|\varepsilon(S)|=1$ that $S / \gamma$ only contains a non-zero idempotent denoted by $e \gamma$. Suppose that there exists $a \in S \backslash E_{S}$. Then $S / \gamma$ must contain a non-idempotent $a \gamma$ from the property that $\operatorname{ker} \gamma=E_{S}$ proved in Lemma 4.1. Since $S / \gamma$ is completely 0 -simple, we have $(a \gamma) \mathcal{L}(a \gamma)^{-1} a \gamma=e \gamma$ and $(a \gamma) \mathcal{R}(a \gamma)(a \gamma)^{-1}=e \gamma$, where $(a \gamma)^{-1}$ is the unique inverse of $a \gamma$ in $S / \gamma$. Hence $(a \gamma) \mathcal{H}(e \gamma)$, which contradicts the fact that $S / \gamma$ is combinatorial, and so $S$ is a 0 -simple band.

The sufficiency follows from Lemmas 3.4 and 4.2 .

Lemma 4.4. For a non-combinatorial completely 0-simple orthodox semigroup $S$, the lattice Subf $S$ is 0-semidistributive if and only if each non-trivial maximal subgroup of $S$ is as described in Lemma 3.2 and $E_{S}$ is a 0-simple band.

Proof. Let $S$ be a non-combinatorial completely 0-simple orthodox semigroup. Then $S$ at least contains a non-trivial maximal subgroup, denoted by $G$, of $S$. It follows from $\operatorname{ker} \gamma=E_{S}$ that $G / \gamma$ is a non-trivial maximal subgroup of $S / \gamma$, and so $S / \gamma$ is not combinatorial. As in the proof of Lemma 4.3, we may show that $S / \gamma$ is a completely 0 -simple inverse semigroup, and so $S$ is a non-combinatorial completely 0 -simple inverse semigroup. Since the lattice Subf $S$ is 0-semidistributive, it follows by Lemma 4.2 that the lattice $\operatorname{Subf}(S / \gamma)$ is 0 -semidistributive. Therefore the lattice $\operatorname{Subfi}(S / \gamma)$ is 0 -semidistributive, and so $\left|E_{S / \gamma}^{*}\right| \leq 2$ by Theorem 2.3 in [16]. On the other hand, since $S / \gamma$ is not combinatorial, we have $\left|E_{S / \gamma}^{*}\right| \neq 2$, and so $\left|E_{S / \gamma}^{*}\right|=1$, that is, $|\varepsilon(S)|=1$, which implies that $E_{S}$ is a 0 -simple band.

It follows from $|\varepsilon(S)|=1$ that $(S / \gamma)^{*}$ is a group. From Lemmas 3.5 and 4.2 we obtain that the lattice $\operatorname{Subf}(S / \gamma)^{*}$ is 0-semidistributive, and so its each non-trivial maximal subgroup is as described in Lemma 3.2. For any non-trivial maximal subgroup $G$ of $S$, it is easy to check that $G \cong G / \gamma$, where $G / \gamma$ is the non-trivial maximal subgroup of $S / \gamma$. Referring to Proposition 2.3.6 of [6], stating that any two group $\mathcal{H}$-classes, which are in the same $\mathcal{D}$-class, are isomorphic, we may conclude that each non-trivial maximal subgroup of $S$ is isomorphic to $G / \gamma$, which is as described in Lemma 3.2 proved above. So the necessity is proved.

The sufficiency follows from Lemmas 3.5 and 4.2 .

As a direct consequence of Lemmas 4.3 and 4.4, we now arrive at the main result in this section.

Theorem 4.5. For an orthodox $G V$ - semigroup $S$, if the lattice Subf $S$ is 0 -semidistributive then

(1) each combinatorial regular $\mathcal{J}$-class, is either a rectangular band or $|\varepsilon(S)|=2$; and

(2) each non-combinatorial regular $\mathcal{J}$-class, whose each non-trivial maximal subgroups is as described in Lemma 3.2 and $E_{J}$ is a rectangular band.

\section{Conclusion}

In the paper, we extend the study of the full subsemigroup lattices of inverse semigroups to that of two particular GV-semigroups. Thus the results and the way in [15] are extended. Inverse semigroups with 0-semidistributive full subsemigroup lattices are first determined. Moreover, the structure of GV-inverse semigroups whose full subsemigroup lattices are 0-semidistributive is characterized. As a consequence, much more explicit structural description is obtained for orthodox GV-semigroups by establishing an 
inverse semigroup congruence. Using the way and the proposed results in the paper, we are to study eventually regular semigroups whose full subsemigroups form certain specific lattices.

Acknowledgments. This research was supported by the the Anhui Natural Science Foundation (No.1308085QA12).

\section{References}

1. Bogdanovic, S., Semigroups with a system of Subsemigroups. Novi Sad University press, 1985.

2. Clifford, A. H. and Preston, G. B., The algebraic theory of semigroups. Amer. Math. Soc, 1976.

3. Edwards, P. M., Eventually regular semigroups. Bull. Austral. Math. Soc, 28 (1983), 23-38.

4. Hall, T. E., On regular semigroups. Journal of algebra, 24 (1973), 1-24.

5. Higgins, P. M., Techniques of semigroup Theory. Oxford university Press, 1992.

6. Howie, J. M., Fundamentals of Semigroup Theory. Clarendon Press, 1995.

7. Johnston, K. G. and Jones, P. R., The lattice of full regular subsemigroups of a regular semigroup. Proc. Royal Soc, 98A (1984), 203-214.

8. Johnston, K. G. and Jones, P. R., Modular inverse semigroups. J. Austral. Math. Soc, 43 (1987), 47-63.

9. Johnston, K. G. and Jones, P. R., Semidistributive inverse semigroups. J. Austral. Math. Soc, 71 (2001), 37-51.

10. Jones, P. R., Distributive inverse semigroups. J. London. Math. Soc, 17 (1978), 457-466.

11. Jones, P. R., Semimodular inverse semigroups. J. London. Math. Soc, 17 (1978), 446-456.

12. Jones, P. R., Inverse semigroups whose full inverse subsemigroups form a chain. Glasgow Math. J, 22 (1981), 159-165.

13. Jones, P. R. Tian, Z. J. and Xu, Z. B., On the lattice of full eventually regular subsemigroups. Communications in algebra, 33 (2005), 2587-2600.

14. Shevrin, L. N. and Ovsyannikov, A. J., Semigroups and their subsemigroup lattices. Kluwer Academic Publishers, 1996.

15. Tian, Z. J., The lattice of full subsemigroups of an inverse semigroup. Semigroup Forum, 73 (2006), 457-469.

16. Tian, Z. J., 0-semidistributive inverse semigroups. Communications in algebra, 35 (2007), 1824-1833.

17. Weipoltshammer, B., On classes of E-inversive semigroups and semigroups whose idempotents form a subsemigroup. Communications in algebra, 32 (2004), 2924-2948.

18. Weipoltshammer, B., Certain congruences on E-inversive E-semigroups. Semigroup Forum, 65 (2002), 233-248. 